\title{
COFINITENESS AND NON-VANISHING OF LOCAL COHOMOLOGY MODULES
}

\author{
IRAJ BAGHERIYEH, KAMAL BAHMANPOUR AND JAFAR A'ZAMI
}

\begin{abstract}
Let $R$ be a commutative Noetherian local ring, $I$ an ideal of $R$, and let $M$ be a non-zero finitely generated $R$-module. In this paper, we establish some new properties of the local cohomology modules $H_{I}^{i}(M), i \geq 0$. In particular, we show that if $(R, \mathfrak{m})$ is a Noetherian local integral domain of dimension $d \leq 4$ which is a homomorphic image of a Cohen-Macaulay ring and $x_{1}, \ldots, x_{n}$ is a part of a system of parameters for $R$, then for all $i \geq 0$, the $R$-modules $H_{I}^{i}(R)$ are $I$-cofinite, where $I=\left(x_{1}, \ldots, x_{n}\right)$. Also, we prove that if $(R, \mathfrak{m})$ is a Noetherian local ring of dimension $d$ and $x_{1}, \ldots, x_{t}$ is a part of a system of parameters for $R$, then $H_{\mathfrak{m}}^{d-t}\left(H_{\left(x_{1}, \ldots, x_{t}\right)}^{t}(R)\right) \neq 0$. In particular, $\mu^{d-t}\left(\mathfrak{m}, H_{\left(x_{1}, \ldots, x_{t}\right)}^{t}(R)\right) \neq 0$ and $\operatorname{injdim}_{R}\left(H_{\left(x_{1}, \ldots, x_{t}\right)}^{t}(R)\right) \geq$ $d-t$.
\end{abstract}

1. Introduction. Throughout this paper, all rings will be assumed to be commutative Noetherian with non-zero identity, and all modules will be assumed to be finitely generated. For each ideal $I$ of a ring $R$ and for each $R$-module $M$, the $i$ th local cohomology module of $M$ with respect to $I$ is defined as

$$
H_{I}^{i}(M)=\underset{n \geq 1}{\lim _{n \geq 1}} \operatorname{Ext}_{R}^{i}\left(R / I^{n}, M\right) .
$$

We refer the reader to $[4,6]$ for more details about local cohomology.

In [8], Huneke asked the following wild problem:

2010 AMS Mathematics subject classification. Primary 13D45, 14B15, Secondary $13 \mathrm{E} 05$.

Keywords and phrases. Associated primes, cofinite modules, Krull dimension, local cohomology.

This research of the authors has been supported by a grant from Islamic Azad University-Hashtroud branch.

Received by the editors on February 3, 2013, and in revised form on June 2, 2013. 
Let $W:=\left\{\operatorname{depth}\left(M_{\mathfrak{p}}\right)+\operatorname{height}(I+\mathfrak{p} / \mathfrak{p}): I \nsubseteq \mathfrak{p} \in \operatorname{Supp} M\right\}$. Is it true that, $0 \leq n \notin W$ if and only if $H_{I}^{n}(M)$ is finitely generated?

Concerning this wild problem, see the interesting paper [11]. In this paper, we obtain a similar result to this problem whenever $R$ is a complete local ring and $I$ is the maximal ideal of $R$.

In 1969, Grothendieck conjectured that, if $I$ is an ideal of $R$ and $M$ is a finitely generated $R$-module, then the $R$-modules $\operatorname{Hom}_{R}\left(R / I, H_{I}^{i}(M)\right)$ are finitely generated for all $i \geq 0$. Hartshorne has provided a counterexample to this conjecture in [7]. Also, he defined a module $T$ to be $I$-cofinite if $\operatorname{Supp} T \subseteq V(I)$ and $\operatorname{Ext}_{R}^{i}(R / I, T)$ is finitely generated for each $i \geq 0$, and he asked the following question.

For which rings $R$ and ideals $I$ are the modules $H_{I}^{i}(M) I$-cofinite for all $i$ and all finitely generated modules $M$ ?

Hartshorne proved that, if $I$ is an ideal of the complete regular local ring $R$ and $M$ a finitely generated $R$-module, then $H_{I}^{i}(M)$ is $I$-cofinite in the two following cases:

(i) $I$ is a principal ideal, (see [7, Corollary 6.3]),

(ii) $I$ is a prime ideal with $\operatorname{dim} R / I=1$, (see [7, Corollary 7.7]).

This subject was studied by several authors afterward, (see $[\mathbf{1}, \mathbf{3}, \mathbf{5}$, $9,13,19])$.

In this paper we also prove some new results concerning the cofinite local cohomology modules and vanishing of certain local cohomology modules.

Recall that, for each $R$-module $M$, all integers $j \geq 0$ and all prime ideals $\mathfrak{p}$ of $R$, the $j$ th Bass number of $M$ with respect to $\mathfrak{p}$ is defined as $\mu^{j}(\mathfrak{p}, M)=\operatorname{dim}_{k(\mathfrak{p})} \operatorname{Ext}_{R_{\mathfrak{p}}}^{j}\left(k(\mathfrak{p}), M_{\mathfrak{p}}\right)$, where $k(\mathfrak{p}):=R_{\mathfrak{p}} / \mathfrak{p} R_{\mathfrak{p}}$. For an Artinian $R$-module $A$ we denote by $\operatorname{Att}_{R} A$ the set of attached prime ideals of $A$. Also, for any ideal $\mathfrak{a}$ of $R$, we denote $\{\mathfrak{p} \in \operatorname{Spec} R: \mathfrak{p} \supseteq \mathfrak{a}\}$ by $V(\mathfrak{a})$. Also, we denote the injective dimension of $M$ by $\operatorname{injdim}_{R}(M)$. For any unexplained notation and terminology, we refer the reader to $[4,14]$.

2. Local cohomology modules over complete local rings. In this section, we study the finiteness properties of local cohomology modules over the complete Noetherian local rings. 
The following theorem is the first main result of this paper.

Theorem 2.1. Let $(R, \mathfrak{m})$ be a complete Noetherian local ring and $I$ an ideal of $R$. Let $M$ be a non-zero finitely generated $R$-module such that $\operatorname{dim} M / I M>0$. Then

$$
\begin{aligned}
& \inf \left\{i \in \mathbf{N}_{0}: H_{\mathfrak{m}}^{i}(M) \text { is not } I \text {-cofinite }\right\} \\
& \quad=\inf \left\{1+\operatorname{depth}\left(M_{\mathfrak{p}}\right): \mathfrak{p} \in \operatorname{Supp} M / I M \text { and } \operatorname{dim} R / \mathfrak{p}=1\right\} \\
& \quad<\infty .
\end{aligned}
$$

Proof. Set

$$
n:=\inf \left\{i \in \mathbf{N}_{0}: H_{\mathfrak{m}}^{i}(M) \text { is not } I \text {-cofinite }\right\},
$$

and

$$
t:=\inf \left\{1+\operatorname{depth}\left(M_{\mathfrak{p}}\right): \mathfrak{p} \in \operatorname{Supp} M / I M \quad \text { and } \quad \operatorname{dim} R / \mathfrak{p}=1\right\} .
$$

First we show that $n<\infty$. To do this, suppose that the contrary is true. Then the $R$-modules $H_{\mathfrak{m}}^{i}(M)$ are $I$-cofinite, for all $i \geq 0$. From the hypothesis, $\operatorname{dim} M / I M>0$, it follows that there exists $\mathfrak{p} \in \operatorname{Supp}(M / I M)$ such that $\operatorname{dim}(R / \mathfrak{p})=1$. So, using [5, Corollary 1] or [17, Corollary 2.5], we deduce that the $R$-modules $H_{\mathfrak{m}}^{i}(M)$ are $\mathfrak{p}$-cofinite, for all $i \geq 0$. But, since $\mathfrak{p} \in \operatorname{Supp}(M / I M) \subseteq \operatorname{Supp}(M)$, it follows from the definition that $M_{\mathfrak{p}} \neq 0$. Therefore, it follows from [4, Theorem 7.3.2] that $H_{\mathfrak{p}}^{\operatorname{dim}_{R_{\mathfrak{p}}}\left(M_{\mathfrak{p}}\right)}(M)_{\mathfrak{p}} \cong H_{\mathfrak{p} R_{\mathfrak{p}}}^{\operatorname{dim}_{R_{\mathfrak{p}}}\left(M_{\mathfrak{p}}\right)}\left(M_{\mathfrak{p}}\right) \neq 0$. Consequently, in view of [2, Lemma 2.1], the $R$-module $H_{\mathfrak{m}}^{1+\operatorname{dim}_{R_{\mathfrak{p}}}\left(M_{\mathfrak{p}}\right)}(M)$ is not $\mathfrak{p}$-cofinite, which is a contradiction. Therefore, we have $n<\infty$. Now, it follows from the assumption that $H_{\mathfrak{m}}^{n}(M)$ is not $I$-cofinite. Consequently, according to Melkersson's theorem [16, Theorem 1.6], there exists $\mathfrak{p}_{1} \in \operatorname{Att} H_{\mathfrak{m}}^{n}(M)$ such that $\operatorname{dim} R /\left(\mathfrak{p}_{1}+I\right) \geqslant 1$, and therefore there is an element $\mathfrak{p} \in V\left(\mathfrak{p}_{1}+I\right)$ such that $\operatorname{dim} R / \mathfrak{p}=1$. Since $0:_{R} M \subseteq 0:_{R} H_{\mathfrak{m}}^{n}(M) \subseteq \mathfrak{p}_{1} \subseteq \mathfrak{p}$, it follows that $\operatorname{dim} M / \mathfrak{p} M=1$. Thus, by [16, Theorem 1.6] and by assumption $\operatorname{dim} R / \mathfrak{p}=1$, we deduce that $H_{\mathfrak{m}}^{n}(M)$ is not $\mathfrak{p}$-cofinite. Hence, by [2, Lemma 2.1], $\mathfrak{p} \in \operatorname{Supp} H_{\mathfrak{p}}^{n-1}(M)$, and so $\operatorname{depth}\left(M_{\mathfrak{p}}\right) \leq n-1$ that implies $t \leq n$. On the other hand, for each $\mathfrak{p} \in \operatorname{Supp} M / I M$ with $\operatorname{dim} R / \mathfrak{p}=1$, it follows from the definition of $n$ and [17, Corollary 2.5] that the $R$-modules $H_{\mathfrak{m}}^{i}(M)$ are $\mathfrak{p}$-cofinite, for $i=0, \ldots, n-1$. Therefore, using [2, Lemma 
2.1] and [4, Theorem 6.2.7], it follows that $\operatorname{depth}\left(M_{\mathfrak{p}}\right) \geq n-1$. Hence, we have $t \geq n$. This completes the proof.

Theorem 2.2. Let $(R, \mathfrak{m})$ be a complete Noetherian local ring and $M$ a non-zero finitely generated $R$-module. Let

$$
W:=\left\{i+1: \mathfrak{p} \in \operatorname{Supp} H_{\mathfrak{p}}^{i}(M) \text { and } \operatorname{dim} R / \mathfrak{p}=1\right\},
$$

and let $n$ be a non-negative integer. Then $H_{\mathfrak{m}}^{n}(M)$ is finitely generated if and only if $n \notin W$.

Proof. Assume that $H_{\mathfrak{m}}^{n}(M)$ is not finitely generated. Then it follows from [4, Corollary 7.2.12] that $\operatorname{Att} H_{\mathfrak{m}}^{n}(M) \nsubseteq\{\mathfrak{m}\}$. Thus, there exists an element $\mathfrak{p}_{1} \in \operatorname{Att} H_{\mathfrak{m}}^{n}(M)$ such that $\operatorname{dim} R / \mathfrak{p}_{1} \geqslant 1$, and so there is an element $\mathfrak{p} \in V\left(\mathfrak{p}_{1}\right)$ such that $\operatorname{dim} R / \mathfrak{p}=1$. Since $0:_{R} M \subseteq 0:_{R} H_{\mathfrak{m}}^{n}(M) \subseteq \mathfrak{p}_{1} \subseteq \mathfrak{p}$, it follows that $\operatorname{dim} M / \mathfrak{p} M=1$. Now, by [16, Theorem 1.6], and by the assumption $\operatorname{dim} R / \mathfrak{p}=1$, we deduce that $H_{\mathfrak{m}}^{n}(M)$ is not $\mathfrak{p}$-cofinite. Hence, by [2, Lemma 2.1], $\mathfrak{p} \in \operatorname{Supp} H_{\mathfrak{p}}^{n-1}(M)$ and so, by definition, $n \in W$. Now let $n \in W$. Then, by definition, there exists $\mathfrak{p} \in \operatorname{Spec}(R) \operatorname{such}$ that $\operatorname{dim} R / \mathfrak{p}=1$ and $\mathfrak{p} \in \operatorname{Supp} H_{\mathfrak{p}}^{n-1}(M)$. Thus, in view of [2, Lemma 2.1], the $R$ module $H_{\mathfrak{m}}^{n}(M)$ is not $\mathfrak{p}$-cofinite and hence is not finitely generated. This completes the proof.

The following result is a generalization of [2, Lemma 2.1].

Proposition 2.3. Let $(R, \mathfrak{m})$ be a complete Noetherian local ring, $I$ an ideal of $R$ and $M$ a finitely generated $R$-module such that $\operatorname{dim} M / I M>$ 0 . Then, for each positive integer $n$, the following statements are equivalent:

(i) $H_{\mathfrak{m}}^{n}(M)$ is I-cofinite.

(ii) For each $\mathfrak{p} \in \operatorname{Supp} M / I M$ with $\operatorname{dim} R / \mathfrak{p}=1, H_{\mathfrak{p} R_{\mathfrak{p}}}^{n-1}\left(M_{\mathfrak{p}}\right)=0$.

(iii) For each $\mathfrak{p} \in \operatorname{Supp} M / I M$ with $\operatorname{dim} R / \mathfrak{p}=1, H_{\mathfrak{p}}^{n-1}(M)$ is Artinian.

Proof. (i) $\rightarrow$ (ii). Let $\mathfrak{p} \in \operatorname{Supp} M / I M$ and $\operatorname{dim} R / \mathfrak{p}=1$. Then, since $H_{\mathfrak{m}}^{n}(M)$ is $I$-cofinite, it follows from [17, Corollary 2.5] that the $R$-modules $H_{\mathfrak{m}}^{n}(M)$ are $\mathfrak{p}$-cofinite. So, the assertion follows from [2, Lemma 2.1]. 
In order to prove (ii) $\rightarrow$ (i), assume that the contrary is true. Then $H_{\mathfrak{m}}^{n}(M)$ is not $I$-cofinite and so, by [16, Theorem 1.6], there exists $\mathfrak{p}_{1} \in \operatorname{Att} H_{\mathfrak{m}}^{n}(M)$ such that $\operatorname{dim} R /\left(\mathfrak{p}_{1}+I\right) \geqslant 1$, and therefore, there is $\mathfrak{p} \in V\left(\mathfrak{p}_{1}+I\right)$ such that $\operatorname{dim} R / \mathfrak{p}=1$. Since $0::_{R} M \subseteq 0:_{R}$ $H_{\mathfrak{m}}^{n}(M) \subseteq \mathfrak{p}_{1} \subseteq \mathfrak{p}$, it follows that $\operatorname{dim} M / \mathfrak{p} M=1$ and $\mathfrak{p} \in \operatorname{Supp} M / I M$. Now, by [16, Theorem 1.6], and by the assumption $\operatorname{dim} R / \mathfrak{p}=1$, we deduce that $H_{\mathfrak{m}}^{n}(M)$ is not $\mathfrak{p}$-cofinite. Hence, by [2, Lemma 2.1], $\mathfrak{p} \in \operatorname{Supp} H_{\mathfrak{p}}^{n-1}(M)$, which is a contradiction. The proof of (ii) $\leftrightarrow$ (iii) follows from [2, Lemma 2.1].

\section{Local cohomology modules over Noetherian local rings.} In this section, we derive some new results about local cohomology modules over Noetherian local rings.

Theorem 3.1. Let $R$ be a Noetherian ring and $M$ an $R$-module. Let $I \subseteq J$ be proper ideals of $R$, and let $t$ be a non-negative integer. Then the following conditions are equivalent:

(i) For all $i \leq t, H_{I}^{i}(M) \cong H_{J}^{i}(M)$.

(ii) For all $i \leq t, \operatorname{Ass}_{R} H_{I}^{i}(M)=\operatorname{Ass}_{R} H_{J}^{i}(M)$.

(iii) For all $i \leq t$, Supp $H_{I}^{i}(M)=\operatorname{Supp} H_{J}^{i}(M)$.

(iv) For all $i \leq t$, Supp $H_{I}^{i}(M) \subseteq V(J)$.

Proof. (i) $\rightarrow$ (ii). This statement is clear.

(ii) $\rightarrow$ (iii). The assertion follows immediately from the fact that, for every $R$-module $T$, we have $\operatorname{Supp} T=\bigcup_{\mathfrak{p} \in \operatorname{AsS}_{R} T} V(\mathfrak{p})$.

The conclusion (iii) $\rightarrow$ (iv) is obvious.

(iv) $\rightarrow$ (i). Since $R$ is Noetherian and $I \subseteq J$, it follows that there exist elements $x_{1}, \ldots, x_{n} \in R$ such that $J=I+R x_{1}+\cdots+R x_{n}$ and $n \geq 0$. We argue by induction on $n$. When $n=0$, we have $I=J$ and so there is nothing to prove. Now suppose, inductively, that $n \geq 1$ and the result has been proved for $n-1$. Then since, for all $i \leq t$, Supp $H_{I}^{i}(M)=\operatorname{Supp} H_{J}^{i}(M) \subseteq V(J) \subseteq V\left(I+R x_{1}+\cdots+R x_{n-1}\right)$, it follows from inductive hypothesis that $H_{I}^{i}(M) \cong H_{I+R x_{1}+\cdots+R x_{n-1}}^{i}(M)$ for $i \leq t$. On the other hand, by assumption, for all $i \leq t$, we have Supp $H_{I}^{i}(M) \subseteq V(J)$, and therefore, $H_{I}^{i}(M)$ is $R x_{n}$-torsion. So, 
$H_{I+R x_{1}+\cdots+R x_{n-1}}^{i}(M)$ is $R x_{n}$-torsion for $i \leq t$. Therefore,

$$
H_{R x_{n}}^{1}\left(H_{I+R x_{1}+\cdots+R x_{n-1}}^{i-1}(M)\right)=0
$$

and

$$
H_{R x_{n}}^{0}\left(H_{I+R x_{1}+\cdots+R x_{n-1}}^{i}(M)\right) \cong H_{I+R x_{1}+\cdots+R x_{n-1}}^{i}(M) \cong H_{I}^{i}(M),
$$

for all $i \leq t$. Consequently, from the exact sequence

$$
\begin{aligned}
0 & \longrightarrow H_{R x_{n}}^{1}\left(H_{I+R x_{1}+\cdots+R x_{n-1}}^{i-1}(M)\right) \\
& \longrightarrow H_{I+R x_{1}+\cdots+R x_{n}}^{i}(M) \\
& \longrightarrow H_{R x_{n}}^{0}\left(H_{I+R x_{1}+\cdots+R x_{n-1}}^{i}(M)\right) \\
& \longrightarrow 0
\end{aligned}
$$

(see [18, Corollary 3.5]), it follows that, for all $i \leq t$,

$$
\begin{aligned}
H_{J}^{i}(M) & =H_{I+R x_{1}+\cdots+R x_{n}}^{i}(M) \cong H_{R x_{n}}^{0}\left(H_{I+R x_{1}+\cdots+R x_{n-1}}^{i}(M)\right) \\
& \cong H_{I}^{i}(M) .
\end{aligned}
$$

This completes the inductive step.

Proposition 3.2. Let $(R, \mathfrak{m})$ be a Cohen-Macaulay Noetherian local ring of dimension $d \geq 1$ and $\mathfrak{p}$ a prime ideal of $R$. Then the following statements are equivalent:

(i) $H_{\mathfrak{m}}^{1}(R / \mathfrak{p})$ is not finitely generated,

(ii) $\operatorname{dim} R / \mathfrak{p}=1$.

Proof. (ii) $\rightarrow$ (i) is clear.

(i) $\rightarrow$ (ii). Since $H_{\mathfrak{m}}^{1}(R / \mathfrak{p})$ is not finitely generated, it follows that $\operatorname{dim} R / \mathfrak{p} \geq 1$. If $\operatorname{dim} R / \mathfrak{p} \geq 2$, then $\mathfrak{p}$ contains an $R$-regular sequence $x_{1}, \ldots, x_{n}$ of length $n=$ height $(\mathfrak{p})$. We have $\operatorname{depth}\left(R /\left(x_{1}, \ldots, x_{n}\right)\right)=\operatorname{dim} R-n=\operatorname{dim} R-\operatorname{height}(\mathfrak{p})=\operatorname{dim} R / \mathfrak{p} \geq 2$ and so $H_{\mathfrak{m}}^{1}\left(R /\left(x_{1}, \ldots, x_{n}\right)\right)=0$. Since height $\left(\left(x_{1}, \ldots, x_{n}\right)\right)=n=$ height $(\mathfrak{p})$ and $\left(x_{1}, \ldots, x_{n}\right) \subseteq \mathfrak{p}$, it follows that $\mathfrak{p}$ is a minimal prime ideal of $\left(x_{1}, \ldots, x_{n}\right)$, and hence, $\mathfrak{p} \in \operatorname{Ass}_{R}\left(R /\left(x_{1}, \ldots, x_{n}\right)\right)$. Therefore, there exists an exact sequence

$$
0 \longrightarrow R / \mathfrak{p} \longrightarrow R /\left(x_{1}, \ldots, x_{n}\right) \longrightarrow T \longrightarrow 0
$$


for some finitely generated $R$-module $T$. This exact sequence implies that $H_{\mathfrak{m}}^{1}(R / \mathfrak{p}) \cong H_{\mathfrak{m}}^{0}(T)$. Since $T$ is finitely generated, it follows that $H_{\mathfrak{m}}^{1}(R / \mathfrak{p})$ is finitely generated, which is a contradiction.

Let $(R, \mathfrak{m})$ be a Noetherian local ring, $I$ a proper ideal of $R$ and $M$ a finitely generated $R$-module such that $\operatorname{Supp} M / I M \nsubseteq V(\mathfrak{m})$. Let $r$ be a non-negative integer such that $H_{I}^{i}(M)$ is Artinian for all $i<r$ and $H_{I}^{r}(M)$ is not Artinian. It is shown in [15, Theorem 3.1] and [10, Theorem 3.10] that $r$ is equal to filter depth, $f$-depth $(I, M)$, of $M$ in $I$, i.e., the length of a maximal filter regular sequence of $M$ in $I$. Recall that we say that a sequence $x_{1}, \ldots, x_{r}$ of elements in the ideal $I$ of the local Noetherian ring $(R, \mathfrak{m})$ is a filter regular sequence for a finitely generated $R$-module $M$, if

$$
x_{i} \notin \mathfrak{p} \text { for all } \mathfrak{p} \in \operatorname{Ass}_{R}\left(M /\left(x_{1}, \ldots, x_{i-1}\right) M\right) \backslash\{\mathfrak{m}\},
$$

for all $i=1, \ldots, r$.

Theorem 3.3. Let $(R, \mathfrak{m})$ be a Noetherian local ring, $M$ a non-zero finitely generated $R$-module of dimension $d \geq 1$ and $0 \leq t \leq d-1$ an integer. Then the following conditions are equivalent:

(i) $H_{\mathfrak{m}}^{t}(M)=0$.

(ii) $\mathfrak{m} \notin \operatorname{Ass}_{R}\left(H_{I}^{t}(M)\right)$, for all ideals $I$ of $R$ with $\operatorname{dim} M / I M \leq 1$.

Proof. (i) $\rightarrow$ (ii). Let $I$ be an ideal of $R$ with $\operatorname{dim} M / I M \leq 1$. If $\operatorname{dim} M / I M=0$, then $H_{I}^{t}(M) \cong H_{\mathfrak{m}}^{t}(M)$ and so there is nothing to prove. Next, let $\operatorname{dim} M / I M=1$. Then there is an element $x$ in $\mathfrak{m}$ such that $\operatorname{dim} M /(I+R x) M=0$ and so in view of [18, Corollary 3.5] there is an exact sequence:

$$
0 \longrightarrow H_{R x}^{1}\left(H_{I}^{t-1}(M)\right) \longrightarrow H_{I+R x}^{t}(M) \longrightarrow H_{R x}^{0}\left(H_{I}^{t}(M)\right) \longrightarrow 0 .
$$

But as $\operatorname{dim} M /(I+R x) M=0$, it follows that the ideal $J:=\operatorname{Ann}_{R}(M)+$ $I+R x$ is an $\mathfrak{m}$-primary ideal. Therefore, using [4, Theorem 4.2.1], it follows that

$$
\begin{aligned}
H_{I+R x}^{t}(M) & \cong H_{\left(I+R x+\operatorname{Ann}_{R}(M)\right) / \operatorname{Ann}_{R}(M)}^{t}(M)=H_{\mathfrak{m} / \operatorname{Ann}_{R}(M)}^{t}(M) \\
& \cong H_{\mathfrak{m}}^{t}(M)=0 .
\end{aligned}
$$

So, from the above exact sequence we can conclude that $H_{R x}^{0}\left(H_{I}^{t}(M)\right)=$ 0 , and hence $H_{\mathfrak{m}}^{0}\left(H_{I}^{t}(M)\right)=0$, which implies that $\mathfrak{m} \notin \operatorname{Ass}_{R}\left(H_{I}^{t}(M)\right)$. 
(ii) $\rightarrow$ (i). The case $t=0$ is clear. So, without loss of generality, we may assume $1 \leq t \leq d-1$. Then we have $d \geq 2$. Since $\operatorname{dim}(M)=d \geq 2$, using the prime avoidance theorem, we can find elements $x_{1}, \ldots, x_{d-1} \in \mathfrak{m}$ such that $x_{1}, \ldots, x_{d-1}$ be a filter regular sequence for $M$. Then $x_{1}, \ldots, x_{d-1}$ is a part of a system of parameters for $M$ (and hence $\left.\operatorname{dim} M /\left(x_{1}, \ldots, x_{d-1}\right) M=1\right)$. Then the $R$-modules

$$
H_{\left(x_{1}, \ldots, x_{d-1}\right)}^{0}(M), H_{\left(x_{1}, \ldots, x_{d-1}\right)}^{1}(M), \ldots, H_{\left(x_{1}, \ldots, x_{d-1}\right)}^{d-2}(M)
$$

are Artinian. But, there is an element $x_{d} \in \mathfrak{m}$, such that $x_{1}, \ldots, x_{d}$ is a system of parameters for $M$. On the other hand, in view of $[\mathbf{1 8}$, Corollary 3.5], there is an exact sequence:

$$
\begin{aligned}
0 & \longrightarrow H_{R x_{d}}^{1}\left(H_{\left(x_{1}, \ldots, x_{d-1}\right)}^{t-1}(M)\right) \longrightarrow H_{\left(x_{1}, \ldots, x_{d}\right)}^{t}(M) \\
& \longrightarrow H_{R x_{d}}^{0}\left(H_{\left(x_{1}, \ldots, x_{d-1}\right)}^{t}(M)\right) \longrightarrow 0
\end{aligned}
$$

Now, as $H_{\left(x_{1}, \ldots, x_{d-1}\right)}^{t-1}(M)$ is Artinian, it follows from Grothendieck's vanishing theorem that

$$
H_{R x_{d}}^{1}\left(H_{\left(x_{1}, \ldots, x_{d-1}\right)}^{t-1}(M)\right)=0 .
$$

Also, since the ideal $\left(x_{1}, \ldots, x_{d}\right)$ is $\mathfrak{m}$-primary, it follows that

$$
H_{\left(x_{1}, \ldots, x_{d}\right)}^{t}(M) \cong H_{\mathfrak{m}}^{t}(M)
$$

As $\operatorname{dim} M /\left(x_{1}, \ldots, x_{d-1}\right) M=1$, by the assumption we have $\mathfrak{m} \notin$ $\operatorname{Ass}_{R}\left(H_{\left(x_{1}, \ldots, x_{d-1}\right)}^{t}(M)\right)$, and so

$$
H_{R x_{d}}^{0}\left(H_{\left(x_{1}, \ldots, x_{d-1}\right)}^{t}(M)\right) \cong H_{\mathfrak{m}}^{0}\left(H_{\left(x_{1}, \ldots, x_{d-1}\right)}^{t}(M)\right)=0 .
$$

Therefore, by the above exact sequence, we have $H_{\mathfrak{m}}^{t}(M)=0$, as required.

Theorem 3.4. Let $(R, \mathfrak{m})$ be a Noetherian local ring and $M$ a nonzero finitely generated $R$-module of dimension $d \geq 1$. Let $0 \leq t \leq d-1$ be an integer such that the $R$-module $H_{\mathfrak{m}}^{t}(M)$ is non-zero and finitely generated. Then, for all ideals $I$ of $R$ with $\operatorname{dim} M / I M \leq 1$, we have $\mathfrak{m} \in \operatorname{Ass}_{R}\left(H_{I}^{t}(M)\right)$. In particular, $H_{I}^{t}(M) \neq 0$.

Proof. Let $I$ be an ideal of $R$ with $\operatorname{dim} M / I M \leq 1$. If $\operatorname{dim} M / I M=$ 0 , then $H_{I}^{t}(M) \cong H_{\mathfrak{m}}^{t}(M)$, and so there is nothing to prove. Now, let $\operatorname{dim} M / I M=1$. Then there is an element $x$ in $\mathfrak{m}$ such that 
$\operatorname{dim} M /(I+R x) M=0$ and so $([\mathbf{1 8}$, Corollary 3.5]) there is an exact sequence:

$$
\begin{gathered}
0 \longrightarrow H_{R x}^{1}\left(H_{I}^{t-1}(M)\right) \longrightarrow H_{I+R x}^{t}(M) \\
\longrightarrow H_{R x}^{0}\left(H_{I}^{t}(M)\right) \longrightarrow 0 . \quad(*)
\end{gathered}
$$

According to the argument given in the proof of Theorem 3.3, we have $H_{I+R x}^{t}(M) \cong H_{\mathfrak{m}}^{t}(M)$. Hence, from the above exact sequence, we can conclude that the $R$-module $H_{R x}^{1}\left(H_{I}^{t-1}(M)\right)$ is finitely generated. But, in view of [4, Theorem 2.2.4], there is an exact sequence

$$
D_{R x}\left(H_{I}^{t-1}(M)\right) \longrightarrow H_{R x}^{1}\left(H_{I}^{t-1}(M)\right) \longrightarrow 0, \quad(* *)
$$

and, in view of [4, Remark 2.2.17], we have $D_{R x}\left(H_{I}^{t-1}(M)\right) \cong$ $\left(H_{I}^{t-1}(M)\right)_{x}$ which implies that $D_{R x}\left(H_{I}^{t-1}(M)\right)=x D_{R x}\left(H_{I}^{t-1}(M)\right)$. Therefore, it follows from the exact sequence $(* *)$ that $H_{R x}^{1}\left(H_{I}^{t-1}(M)\right)=$ $x H_{R x}^{1}\left(H_{I}^{t-1}(M)\right)$. Now it follows from Nakayama's lemma that

$$
H_{R x}^{1}\left(H_{I}^{t-1}(M)\right)=0 .
$$

Consequently, it follows from the exact sequence $(*)$,

$$
H_{\mathfrak{m}}^{0}\left(H_{I}^{t}(M)\right) \cong H_{R x}^{0}\left(H_{I}^{t}(M)\right) \neq 0,
$$

which implies that $\mathfrak{m} \in \operatorname{Ass}_{R}\left(H_{I}^{t}(M)\right)$, as required.

Now we present a new and short proof of Grothendieck's nonvanishing theorem. In the proof of this theorem we do not need to reduce to the complete regular local ring case.

Theorem 3.5. Let $(R, \mathfrak{m})$ be a Noetherian local ring, and let $M$ be a non-zero finitely generated $R$-module of dimension $n$. Then $H_{\mathfrak{m}}^{n}(M) \neq$ 0 .

Proof. We argue by induction on $\operatorname{dim} M=n$. When $n=0$, we have $H_{\mathfrak{m}}^{0}(M) \cong M \neq 0$. Now suppose that $n>0$ and the case $n-1$ is settled. Then there is an element $\mathfrak{p} \in \operatorname{Supp} M$ such that $\operatorname{dim}_{R_{\mathfrak{p}}} M_{\mathfrak{p}}=n-1$ and $\operatorname{dim} M / \mathfrak{p} M=1$. Next, by the inductive hypothesis, we have $H_{\mathfrak{p} R_{\mathfrak{p}}}^{n-1}\left(M_{\mathfrak{p}}\right) \neq 0$, and so by [2, Lemma 2.1], $H_{\mathfrak{m}}^{n}(M)$ is not $\mathfrak{p}$-cofinite; therefore, $H_{\mathfrak{m}}^{n}(M)$ is not finitely generated $R$-module. Hence, $H_{\mathfrak{m}}^{n}(M) \neq 0$. The inductive step is now complete. 
Before bringing the next results, note that, in [13, Theorem 3.4], it is shown that if $(R, \mathfrak{m})$ is a Noetherian local ring, $I$ is an ideal of $R$ with $\operatorname{dim} R / I=2$ and $M$ is a finitely generated $R$-module of dimension $d$, then the Bass numbers $\mu^{j}\left(\mathfrak{p}, H_{I}^{i}(M)\right)$ are finite for all $i, j$ and all but finitely many primes $\mathfrak{p}$. The following result shows that, in some special cases, all Bass numbers of the local cohomology module $H_{I}^{d-1}(M)$ are finite.

Theorem 3.6. Let $(R, \mathfrak{m})$ be a Noetherian local ring, $I$ an ideal of $R$ with $\operatorname{dim} R / I=2$, and $M$ a finitely generated $R$-module of dimension $d \geq 2$ such that $\operatorname{Supp} H_{I}^{d-2}(M) \subseteq\{\mathfrak{m}\}$. Then, for any ideal $J$ of $R$ with $I \subseteq J \subseteq \mathfrak{m}$ and $\operatorname{dim} R / J \leq 1$, the $R$-modules $\operatorname{Ext}_{R}^{j}\left(R / J, H_{I}^{d-1}(M)\right)$ are finitely generated for all $j \geq 0$.

Proof. From $\operatorname{dim} R / J \leq 1$, it follows that there exists an element $x \in J$ such that

$$
\operatorname{dim} R /(I+R x)=1 \text {. }
$$

Next, let $L:=I+R x$. Then, in view of [18, Corollary 3.5], there are the following exact sequences:

$$
\begin{aligned}
0 & \longrightarrow H_{R x}^{1}\left(H_{I}^{d-2}(M)\right) \longrightarrow H_{L}^{d-1}(M) \\
& \longrightarrow H_{R x}^{0}\left(H_{I}^{d-1}(M)\right) \longrightarrow 0, \quad(*)
\end{aligned}
$$

and

$$
0 \longrightarrow H_{R x}^{1}\left(H_{I}^{d-1}(M)\right) \longrightarrow H_{L}^{d}(M) \longrightarrow H_{R x}^{0}\left(H_{I}^{d}(M)\right) \longrightarrow 0 .
$$

But, according to the hypothesis, we have $\operatorname{Supp} H_{I}^{d-2}(M) \subseteq\{\mathfrak{m}\}$, and hence the $R$-module $H_{I}^{d-2}(M)$ is $R x$-torsion implying that

$$
H_{R x}^{1}\left(H_{I}^{d-2}(M)\right)=0 .
$$

Therefore, from the exact sequence $(*)$, we get the following isomorphism:

$$
H_{L}^{d-1}(M)=H_{R x}^{0}\left(H_{I}^{d-1}(M)\right) .
$$

Now, as $\operatorname{dim} R / L=1$, according to the main result of [5], or in view of [3, Corollary 2.7], the $R$-module $H_{L}^{d-1}(M)$ is $L$-cofinite. So the $R$-module

$$
H_{R x}^{0}\left(H_{I}^{d-1}(M)\right)=H_{L}^{0}\left(H_{I}^{d-1}(M)\right)
$$


is $L$-cofinite. On the other hand, by [4, Exercise 2.1.9], we have

$$
H_{R x}^{1}\left(H_{I}^{d-1}(M)\right)=H_{L}^{1}\left(H_{I}^{d-1}(M)\right) .
$$

Moreover, by [17, Proposition 5.1], the $R$-module $H_{L}^{d}(M)$ is Artinian and $L$-cofinite. So, using [17, Corollary 4.4], it follows from the exact sequence $(* *)$, that the $R$-module

$$
H_{R x}^{1}\left(H_{I}^{d-1}(M)\right)=H_{L}^{1}\left(H_{I}^{d-1}(M)\right),
$$

is $L$-cofinite. Thus, the $R$-modules $H_{L}^{0}\left(H_{I}^{d-1}(M)\right.$ and $H_{L}^{1}\left(H_{I}^{d-1}(M)\right)$ are $L$-cofinite. But, in view of [12, Corollary 2.5], the set Supp $H_{I}^{d-1}(M)$ is finite and $\operatorname{dim} H_{I}^{d-1}(M) \leq 1$. So, it follows from Grothendieck's vanishing theorem, that

$$
H_{L}^{j}\left(H_{I}^{d-1}(M)\right)=0,
$$

for all $j \geq 2$. Consequently, by [17, Proposition 3.9] for all $j \geq 0$, the $R$-modules $\operatorname{Ext}_{R}^{j}\left(R / L, H_{I}^{d-1}(M)\right)$ are finitely generated. As $L \subseteq J$, it follows from [5, Corollary 1] or [17, Corollary 2.5] that, for all $j \geq 0$, the $R$-modules $\operatorname{Ext}_{R}^{j}\left(R / J, H_{I}^{d-1}(M)\right)$ are finitely generated, as required.

Corollary 3.7. Let $R, I$ and $M$ be as in Theorem 3.6. Then the Bass numbers of the $R$-module $H_{I}^{d-1}(M)$ are finite.

Proof. In view of [12, Corollary 2.5], the set Supp $H_{I}^{d-1}(M)$ is finite and $\operatorname{dim} H_{I}^{d-1}(M) \leq 1$. Hence, for any $\mathfrak{p} \in \operatorname{Supp} H_{I}^{d-1}(M)$, we have $I \subseteq \mathfrak{p} \subseteq \mathfrak{m}$ and $\operatorname{dim} R / \mathfrak{p} \leq 1$. So, by Theorem 3.6, the $R$-modules $\operatorname{Ext}_{R}^{j}\left(R / \mathfrak{p}, H_{I}^{d-1}(M)\right)$ are finitely generated for all $j \geq 0$.

Theorem 3.8. Let $(R, \mathfrak{m})$ be a Noetherian local ring, $I$ an ideal of $R$ with $\operatorname{dim} R / I=2$ and $M$ a finitely generated $R$-module of $d i$ mension $d \geq 2$, such that $\operatorname{dim} H_{I}^{d-2}(M) \leq 1$. Then the $R$-modules $\operatorname{Ext}_{R}^{j}\left(R / \mathfrak{m}, H_{I}^{d-1}(M)\right)$, are finitely generated for all $j \geq 0$. In particular, the Bass numbers of the $R$-module $H_{I}^{d-1}(M)$ are finite.

Proof. By Theorem 3.6, we may assume that $\operatorname{dim} H_{I}^{d-2}(M)=$ 1. Since $\operatorname{dim} R / I=2$, it follows from [3, Corollary 3.3], that 
$\operatorname{Ass}_{R} H_{I}^{d-2}(M)$ is a finite set. Next, suppose that

$$
\operatorname{Ass}_{R} H_{I}^{d-2}(M) \backslash\{\mathfrak{m}\}=\left\{\mathfrak{p}_{1}, \ldots, \mathfrak{p}_{s}\right\},
$$

and

$$
\operatorname{Assh}_{R}(R / I)=\left\{\mathfrak{q}_{1}, \ldots, \mathfrak{q}_{t}\right\} .
$$

Now, by the prime avoidance theorem, there exists an element $x$ in $\mathfrak{m}$, such that $x \notin \bigcup_{i=1}^{s} \mathfrak{p}_{i}$ and $x \notin \bigcup_{i=1}^{t} \mathfrak{q}_{i}$. Whence, $\operatorname{dim} R /(I+R x)=1$ and Supp $H_{R x}^{1}\left(H_{I}^{d-2}(M)\right) \subseteq\{\mathfrak{m}\}$. Let $L:=I+R x$. Then, in view of [18, Corollary 3.5], there are exact sequences:

$0 \longrightarrow H_{R x}^{1}\left(H_{I}^{d-2}(M)\right) \longrightarrow H_{L}^{d-1}(M) \longrightarrow H_{R x}^{0}\left(H_{I}^{d-1}(M)\right) \longrightarrow 0$,

and

$$
0 \longrightarrow H_{R x}^{1}\left(H_{I}^{d-1}(M)\right) \longrightarrow H_{L}^{d}(M) \longrightarrow H_{R x}^{0}\left(H_{I}^{d}(M)\right) \longrightarrow 0 .
$$

Applying the method used in the proof of Theorem 3.6, we have

$$
\begin{aligned}
& H_{R x}^{0}\left(H_{I}^{d-1}(M)\right)=H_{L}^{0}\left(H_{I}^{d-1}(M)\right), \\
& H_{R x}^{1}\left(H_{I}^{d-1}(M)\right)=H_{L}^{1}\left(H_{I}^{d-1}(M)\right),
\end{aligned}
$$

and

$$
H_{L}^{j}\left(H_{I}^{d-1}(M)\right)=0, \quad \text { for all } j \geq 2 .
$$

On the other hand, according to the [3, Corollary 2.7], the $R$-module $H_{L}^{d-1}(M)$ is $L$-cofinite. Therefore, it follows from the exact sequence $(*)$, that the $R$-module $\operatorname{Hom}_{R}\left(R / L, H_{R x}^{1}\left(H_{I}^{d-2}(M)\right)\right)$ is finitely generated. But, we have

$$
\operatorname{Supp} H_{R x}^{1}\left(H_{I}^{d-2}(M)\right) \subseteq\{\mathfrak{m}\},
$$

and hence the $R$-module

$$
\operatorname{Hom}_{R}\left(R / L, H_{R x}^{1}\left(H_{I}^{d-2}(M)\right)\right),
$$

has support in $V(\mathfrak{m})$. Whence, we can deduce that the $R$-module

$$
\operatorname{Hom}_{R}\left(R / L, H_{R x}^{1}\left(H_{I}^{d-2}(M)\right)\right),
$$

is of finite length. Consequently, it follows from [17, Proposition 4.1] that the $R$-module $H_{R x}^{1}\left(H_{I}^{d-2}(M)\right)$ is Artinian and $L$-cofinite. Thus, the $R$-module $H_{L}^{0}\left(H_{I}^{d-1}(M)\right)$ is also $L$-cofinite. On the other hand, applying the method used in the proof of Theorem 3.6, we can see 
that the $R$-module $H_{L}^{1}\left(H_{I}^{d-1}(M)\right)$ is $L$-cofinite, too. Now, by $[\mathbf{1 7}$, Proposition 3.9] for all $j \geq 0$, the $R$-modules $\operatorname{Ext}_{R}^{j}\left(R / L, H_{I}^{d-1}(M)\right)$ are finitely generated. Therefore, as in the proof of Theorem 3.6, since $L \subseteq$ $\mathfrak{m}$, it follows that, for all $j \geq 0$, the $R$-modules $\operatorname{Ext}_{R}^{j}\left(R / \mathfrak{m}, H_{I}^{d-1}(M)\right)$ are finitely generated. On the other hand, if $\mathfrak{m} \neq \mathfrak{p} \in \operatorname{Supp}\left(H_{I}^{d-1}(M)\right)$, then as $\operatorname{dim}\left((M / I M)_{\mathfrak{p}}\right) \leq 1$, it follows from [3, Corollary 2.10] that the Bass numbers of the $R$-module $H_{I}^{d-1}(M)$ with respect to $\mathfrak{p}$ are finite. This completes the proof.

\section{Cofiniteness of local cohomology modules for ideals gen- erated by a part of a system of parameters.}

Theorem 4.1. Let $R$ be a Noetherian ring, $I$ an ideal of $R$ and $M a$ finitely generated $R$-module. Let $s$ be a non-negative integer such that, for all $i \neq s$, the $R$-module $H_{I}^{i}(R)$ is $I$-cofinite. Then $H_{I}^{s}(R)$ is also I-cofinite.

Proof. See [13, Proposition 2.5].

Theorem 4.2. Let $(R, \mathfrak{m})$ be a Noetherian local ring of dimension $d \geq 1$ and $I$ an ideal of $R$ such that $\operatorname{dim} R / I=d$. Let $a_{1}, \ldots, a_{d} \in \mathfrak{m}$ be system of parameters for $R$-module $R / I$. Then there are elements $b_{1}, \ldots, b_{d} \in I$ such that the elements $c_{1}:=a_{1}+b_{1}, \ldots, c_{d}:=a_{d}+b_{d}$ compose a system of parameters for $R$.

Proof. Since $\operatorname{dim}\left(R /\left(I+R a_{1}\right)\right)=d-1$, it follows that $I+R a_{1} \nsubseteq$ $\bigcup_{\mathfrak{p} \in \operatorname{Assh}_{R}(R)} \mathfrak{p}$. So, in view of [14, Exercise 16.8], there exists an element $b_{1} \in I$ such that $b_{1}+a_{1} \notin \bigcup_{\mathfrak{p} \in \operatorname{Assh}_{R}(R)} \mathfrak{p}$. Now $c_{1}:=a_{1}+b_{1}$ is a part of a system of parameters for $R$. Let, by induction, the elements $c_{1}=a_{1}+b_{1}, \ldots, c_{k}=a_{k}+b_{k}$ be a part of a system of parameters for $R$, where $1 \leq k \leq d-1$ and $\left\{b_{1}, \ldots, b_{k}\right\} \subseteq I$. Then we have

$$
I+R a_{1}+\cdots+R a_{k}=I+R c_{1}+\cdots+R c_{k}
$$

and

$$
\operatorname{dim}\left(R /\left(I+R a_{1}+\cdots+R a_{k}+R a_{k+1}\right)\right)=d-(k+1) .
$$

Consequently, $\left(I+R c_{1}+\cdots+R c_{k}+R a_{k+1}\right) \nsubseteq \bigcup_{\mathfrak{p} \in \operatorname{Assh}_{R}\left(R /\left(c_{1}, \ldots, c_{k}\right)\right)} \mathfrak{p}$. Since $\left(c_{1}, \ldots, c_{k}\right) \subseteq \bigcap_{\mathfrak{p} \in \operatorname{Assh}_{R}\left(R /\left(c_{1}, \ldots, c_{k}\right)\right)} \mathfrak{p}$, it follows that $I+R a_{k+1} \nsubseteq$ 
$\bigcup_{\mathfrak{p} \in \operatorname{Assh}_{R}\left(R /\left(c_{1}, \ldots, c_{k}\right)\right)} \mathfrak{p}$. Therefore, in view of [14, Exercise 16.8], there exists $b_{k+1} \in I$ such that $a_{k+1}+b_{k+1} \notin \bigcup_{\mathfrak{p} \in \operatorname{Assh}_{R}\left(R /\left(c_{1}, \ldots, c_{k}\right)\right)} \mathfrak{p}$. Set $c_{k+1}:=a_{k+1}+b_{k+1}$. Then it is easy to see that $c_{1}, \ldots, c_{k+1}$ is a part of a system of parameters for $R$. This completes the inductive step.

Theorem 4.3. Let $(R, \mathfrak{m})$ be a Noetherian local ring of dimension $d=3$ and $x_{1}, x_{2}, x_{3}$ a system of parameters for $R$. Set $I_{1}=\left(x_{1}\right)$, $I_{2}=\left(x_{1}, x_{2}\right)$ and $I_{3}=\left(x_{1}, x_{2}, x_{3}\right)$. Then, for each $1 \leq n \leq 3$ and each $i \geq 0$, the $R$-module $H_{I_{n}}^{i}(R)$ is $I_{n}$-cofinite.

Proof. In the case $n=1$, the ideal $I_{n}$ is principal and so the result is well known. The cases $n=2,3$ follow from the main result of [5].

Theorem 4.4. Let $(R, \mathfrak{m})$ be a Noetherian local integral domain of dimension $d \leq 4$ which is a homomorphic image of a Cohen-Macaulay local ring. Let $1 \leq n \leq 4$ and $x_{1}, \ldots, x_{n}$ be a part of a system of parameters for $R$. Then, for each $i \geq 0$, the $R$-module $H_{I}^{i}(R)$ is a $I$-cofinite, where $I=\left(x_{1}, \ldots, x_{n}\right)$.

Proof. Let $(R, \mathfrak{m})$ be a homomorphic image of the Cohen-Macaulay $\operatorname{ring}(S, \mathfrak{n})$ with $\operatorname{dim} S=d_{1}$. Then $d_{1} \geq d$. We may assume that $R=S / J$ for some ideal $J$ of $S$. Then we have height $(J)=d_{1}-d$, and so there exists an $R$-sequence as $y_{1}, \ldots, y_{d_{1}-d}$ contained in $J$. Now we can replace the ring $S$ with the Cohen-Macaulay local ring $T:=S /\left(y_{1}, \ldots, y_{d_{1}-d}\right)$. So, we may assume that $n=2$. Since $R$ is an integral domain and $\operatorname{dim} R=\operatorname{dim} S$ it follows that $R=S / \mathfrak{p}$ for some $\mathfrak{p} \in \operatorname{Assh}_{S}(S)$ and so there is an exact sequence

$$
0 \longrightarrow R \longrightarrow S \longrightarrow K
$$

for some finitely generated $S$-module $K$. Since $x_{1}, x_{2} \in \mathfrak{m}=\mathfrak{n} / \mathfrak{p}$, it follows that $x_{1}=t_{1}+\mathfrak{p}$ and $x_{2}=t_{2}+\mathfrak{p}$, for some elements $t_{1}, t_{2} \in \mathfrak{n}$. Now, by Lemma 4.1 , there exist elements $y_{1}, y_{2} \in \mathfrak{p}$ such that $z_{1}:=t_{1}+y_{1}$ and $z_{2}:=t_{2}+y_{2}$ is a part of a system of parameters for $S$. Therefore, as $S$ is a Cohen-Macaulay ring, it follows that $z_{1}, z_{2}$ is an $S$-sequence and, hence, according to [4, Theorem 6.2.7] we have

$$
H_{\left(z_{1}, z_{2}\right)}^{0}(S)=0=H_{\left(z_{1}, z_{2}\right)}^{1}(S)=0 .
$$


So, from the exact sequence $(*)$, we get the following isomorphism:

$$
H_{\left(z_{1}, z_{2}\right)}^{1}(T) \cong H_{\left(z_{1}, z_{2}\right)}^{1}(R) \text {. }
$$

But, using [4, Theorem 4.2.1] we have

$$
H_{\left(z_{1}, z_{2}\right)}^{1}(R)=H_{\left(z_{1}, z_{2}\right)+\mathfrak{p} / \mathfrak{p}}^{1}(R)=H_{\left(t_{1}, t_{2}\right)+\mathfrak{p} / \mathfrak{p}}^{1}(R)=H_{\left(x_{1}, x_{2}\right)}^{1}(R) .
$$

Since $H_{\left(z_{1}, z_{2}\right)}^{0}(T)$ is a finitely generated $S$-module, it follows that $H_{\left(x_{1}, x_{2}\right)}^{1}(R)$ is a finitely generated $S$-module, and so $H_{\left(x_{1}, x_{2}\right)}^{1}(R)$ is a finitely generated $R$-module. In particular, $H_{\left(x_{1}, x_{2}\right)}^{1}(R)$ is $\left(x_{1}, x_{2}\right)$ cofinite. On the other hand, in view of [4, Theorem 3.3.1], for $i \geq 3$, we have $H_{\left(x_{1}, x_{2}\right)}^{i}(R)=0$. Now, by [13, Proposition 2.5], the $R$-module $H_{\left(x_{1}, x_{2}\right)}^{2}(R)$ is $\left(x_{1}, x_{2}\right)$-cofinite. This completes the proof.

Theorem 4.5. Let $(R, \mathfrak{m})$ be a Noetherian local ring of dimension $d$ and $x_{1}, \ldots, x_{n}$ a part of a system of parameters for $R$. Then $H_{\mathfrak{m}}^{d-t}\left(H_{\left(x_{1}, \ldots, x_{t}\right)}^{t}(R)\right) \neq 0$. In particular, $\mu^{d-t}\left(\mathfrak{m}, H_{\left(x_{1}, \ldots, x_{t}\right)}^{t}(R)\right) \neq 0$ and $\operatorname{injdim}_{R}\left(H_{\left(x_{1}, \ldots, x_{t}\right)}^{t}(R)\right) \geq d-t$.

Proof. Let $x_{1}, \ldots, x_{t}, \ldots, x_{d}$ be a system of parameters for $R$. Then, using [18, Corollary 3.5] and [4, Theorem 3.3.1], we have the following:

$$
\begin{gathered}
H_{R x_{t+1}}^{1}\left(H_{\left(x_{1}, \ldots, x_{t}\right)}^{t}(R)\right) \cong H_{\left(x_{1}, \ldots, x_{t+1}\right)}^{t+1}(R) \\
H_{R x_{t+2}}^{1}\left(H_{\left(x_{1}, \ldots, x_{t+1}\right)}^{t+1}(R)\right) \cong H_{\left(x_{1}, \ldots, x_{t+2}\right)}^{t+2}(R) \cong H_{\left(x_{t+1}, x_{t+2}\right)}^{2}\left(H_{\left(x_{1}, \ldots, x_{t}\right)}^{t}(R)\right) \\
\vdots \\
H_{R x_{d}}^{1}\left(H_{\left(x_{1}, \ldots, x_{d-1}\right)}^{d-1}(R)\right) \cong H_{\left(x_{1}, \ldots, x_{d}\right)}^{d}(R) \\
\cong H_{\left(x_{t+1}, x_{t+2}, \ldots, x_{d}\right)}^{d-t}\left(H_{\left(x_{1}, \ldots, x_{t}\right)}^{t}(R)\right)
\end{gathered}
$$

But, by Grothendieck's non-vanishing theorem, we have $H_{\left(x_{1}, \ldots, x_{d}\right)}^{d}(R)$ $=H_{\mathfrak{m}}^{d}(R) \neq 0$. Therefore, it follows from [4, Exercise 2.1.9] that

$$
\begin{aligned}
H_{\mathfrak{m}}^{d-t}\left(H_{\left(x_{1}, \ldots, x_{t}\right)}^{t}(R)\right) & \cong H_{\left(x_{1}, \ldots, x_{d}\right)}^{d-t}\left(H_{\left(x_{1}, \ldots, x_{t}\right)}^{t}(R)\right) \\
& \cong H_{\left(x_{t+1}, x_{t+2}, \ldots, x_{d}\right)}^{d-t}\left(H_{\left(x_{1}, \ldots, x_{t}\right)}^{t}(R)\right) \neq 0
\end{aligned}
$$

as required. 
Acknowledgments. The authors are deeply grateful to the referee for a very careful reading of the manuscript and many valuable suggestions. Also we would like to thank the Islamic Azad UniversityHashtroud branch for financial support.

\section{REFERENCES}

1. R. Abazari and K. Bahmanpour, Cofiniteness of extension functors of cofinite modules, J. Algebra 330 (2011), 507-516.

2. I. Bagheriyeh, J. A'zami and K. Bahmanpour, Generalization of the Lichtenbaum-Hartshorne vanishing theorem, Comm. Algebra 40 (2012), 134-137.

3. K. Bahmanpour and R. Naghipour, Cofiniteness of local cohomology modules for ideals of small dimension, J. Algebra 321 (2009), 1997-2011.

4. M.P. Brodmann and R.Y. Sharp, Local cohomology; An algebraic introduction with geometric applications, Cambridge University Press, Cambridge, 1998.

5. D. Delfino and T. Marley, Cofinite modules and local cohomology, J. Pure Appl. Alg. 121 (1997), 45-52.

6. A. Grothendieck, Local cohomology, Lect. Notes Math. 862, Springer, New York, 1966.

7. R. Hartshorne, Affine duality and cofiniteness, Inv. Math. 9 (1970), 145-164.

8. C. Huneke, Problems on local cohomology, Free resolutions in commutative algebra and algebraic geometry, Res. Notes Math. 2 (1992), 93-108.

9. K.-I. Kawasaki, On the finiteness of Bass numbers of local cohomology modules, Proc. Amer. Math. Soc. 124 (1996), 3275-3279.

10. R. Lü and Z. Tang, The f-depth of an ideal on a module, Proc. Amer. Math. Soc. 130 (2001), 1905-1912.

11. T. Marley, Finitely graded local cohomology and depths of graded algebra, Proc. Amer. Math. Soc. 123 (1995), 3601-3607.

12. , The associated primes of local cohomology modules over rings of small dimension, Manuscr. Math. 104 (2001), 519-525.

13. T. Marley and J.C. Vassilev, Cofiniteness and associated primes of local cohomology modules, J. Algebra 256 (2002), 180-193.

14. H. Matsumura, Commutative ring theory, Cambridge University Press, Cambridge, UK, 1986.

15. L. Melkersson, On asymptotic stability for sets of prime ideals connected with the powers of an ideal, Math. Proc. Cambr. Phil. Soc. 107 (1990), 267-271.

16. L. Melkersson, Properties of cofinite modules and application to local cohomology, Math. Proc. Cambr. Phil. Soc. 125 (1999), 417-423.

17. T. Melkersson, Modules cofinite with respect to an ideal, J. Algebra 285 (2005), 649-668.

18. P. Schenzel, Proregular sequences, local cohomology, and completion, Math. Scand. 92 (2003), 161-180. 
19. K.I. Yoshida, Cofiniteness of local cohomology modules for ideals of dimension one, Nagoya Math. J. 147 (1997), 179-191.

Department of Mathematics, Islamic Azad University-Hashtroud Branch, HASHTROUd, IRAN

Email address: Ir_ba2004@yahoo.com

Department of Mathematics, Faculty of Mathematical Sciences, UniverSity of Mohaghegh Ardabili, 56199-11367, Ardabil, Iran

Email address: bahmanpour.k@gmail.com

Department of Mathematics, Faculty of Mathematical Sciences, UniverSity of Mohaghegh Ardabili, 56199-11367, Ardabil, Iran

Email address: jafar.azami@gmail.com, azami@uma.ac.ir 